\title{
Role of Colour Doppler in Evaluation of Intrauterine Growth Retardation
}

\author{
Manoj Kumar Veerabathini ${ }^{1}$, Sudhanshu Sekhar Mohanthy ${ }^{2}$, Nilanjan Mukherjee ${ }^{3}$, Aavula Adarsh ${ }^{4}$, B Arun \\ Kumar $^{5}$, Girendra Shankar 6 \\ ${ }^{1}$ Junior Resident, Department of Radio-Diagnosis, ${ }^{2}$ Assistant Professor, Department of Radio-Diagnosis, ${ }^{3}$ Junior Resident, \\ Department of Radio-Diagnosis, 4 Junior Resident, Department of Radio-Diagnosis, 5Junior Resident, Department of Radio- \\ Diagnosis, ${ }^{6} J u n i o r$ Resident, Department of Radio-Diagnosis, Kalinga Institute of Medical sciences, Campus No:5, KIIT Road, \\ Patia, Bhubaneswar, Odisha-751024, India
}

Corresponding author: Dr Sudhanshu Sekhar Mohanthy, Assistant Professor, Department of Radio-Diagnosis, Kalinga Institute of Medical Sciences, Campus No:5, KIIT Road, Patia, Bhubaneswar, Odisha-751024, India

DOI: http://dx.doi.org/10.21276/ijcmsr.2020.5.1.32

BY-NC-ND

How to cite this article: Manoj Kumar Veerabathini, Sudhanshu Sekhar Mohanthy, Nilanjan Mukherjee, Aavula Adarsh, B Arun Kumar, Girendra Shankar. Role of colour doppler in evaluation of intrauterine growth retardation. International Journal of Contemporary Medicine Surgery and Radiology. 2020;5(1):A148-A152.

\section{A B S T R A C T}

Introduction: Most common complication of pregnancy is intrauterine growth restriction which is associated with perinatal mortality, morbidity and neurological impaired development. Doppler of Uterine, umbilical, middle cerebral arteries and cerebro-placental ratio are commonly done for intrauterine growth restriction evaluation. The aim of the study was to evaluate the diagnostic performance of fetal middle cerebral artery and umbilical artery by Doppler ultrasonography in high risk mothers and correlation with perinatal outcome.

Material and Methods: Fifty singleton pregnancies beyond 28 weeks of gestation complicated by intrauterine growth restriction were prospectively examined with Doppler ultrasound of umbilical and middle cerebral arteries.

Results: $\mathbf{4 0}$ of the 50 cases included in the study had adverse outcome. The sensitivity of Doppler of the umbilical artery using Pulsatility index was $82.5 \%$, middle cerebral artery Pulsatility index was $77.5 \%$ and ratio of middle cerebral artery Pulsatility index / umbilical artery Pulsatility index was $87.5 \%$ in predicting perinatal outcome.

Conclusion: Pulsatility index ratio of middle cerebral artery / umbilical artery is the most sensitive index in predicting perinatal outcome (87.5\%). Umbilical Systolic /Diastolic ratio is the most specific index (80.9\%). Absent and reversed diastolic flow in umbilical artery in prenatal sonography carries grave prognosis and high mortality of fetus. Combined use of both umbilical and middle cerebral arterial doppler study including the cerebro-placental ratio increase the sensitivity of the doppler studies for prediction adverse perinatal outcome.

Keywords: Colour Doppler, Intrauterine Growth Retardation

\section{INTRODUCTION}

Intrauterine growth restriction (IUGR) is defined as sonographic estimated fetal weight less than the 10th percentile for gestational age, whereas small for gestational age (SGA) will be used exclusively to describe newborns whose birth weight is less than the 10th percentile for gestational age. ${ }^{1,2}$ Low birth weight (LBW) is defined as birth weight less than 2,500 $\mathrm{g}$ and should not be confused with IUGR/SGA, as the definition of LBW is based on the irrespective of gestational age, sex, race, and clinical features. ${ }^{3}$ The prevalence of low birth weight baby is $33 \%$.

The incidence of IUGR is more in developing countries (6$30 \%$ ) than developed countries (4-8\%). The average incidence of IUGR is about $8 \%$ in the general population. More than $50 \%$ of stillbirth baby are associated with IUGR and about $10 \%$ of perinatal mortality is consequent to undetected IUGR. Placental insufficiency, maternal hypertension, diabetes, infection, low-socioeconomic status and preeclampsia are common risk factors for IUGR. Most common complication of pregnancy is IUGR which is associated with perinatal mortality, morbidity and neurological impaired development. ${ }^{4}$ Fetal and maternal doppler ultrasound helps in detection of compromised IUGR fetus by doing timely intervention which is main objective of antenatal care. ${ }^{5}$

IUGR are mainly due to increased resistance within the placental circulation which leads to decrease in velocity in the feeding arteries especially during diastole than systole. Disproportionate slowing of diastolic relative to systolic flow leads to elevation of a number of Doppler indices, including the systolic/diastolic ratio and the pulsatility index. ${ }^{6}$ Uterine artery Doppler, umbilical artery Doppler, middle cerebral artery Doppler, cerebro-placental ratio (CPR), ductus venosus Doppler, and aortic isthmus Doppler are commonly done for IUGR evaluation. ${ }^{7}$ Uterine arteries provide knowledge of maternal circulation, whereas the umbilical and middle cerebral arteries give information about the fetal circulation. Uterine artery Doppler has limited sensitivity 
and specificity to predict adverse outcome in IUGR fetuses diagnosed during the third trimester. ${ }^{8}$ The utility of umbilical artery Doppler velocimetry has been extensively reviewed in cases of fetal growth restriction. Absent or reversed enddiastolic flow in the umbilical artery is associated with an increased risk of perinatal mortality.

The purpose of this study was to evaluate the diagnostic performance of fetal middle cerebral artery (MCA) and umbilical artery (UA) by Doppler ultrasonography in high risk mothers with IUGR and correlation with perinatal outcome.

\section{MATERIAL AND METHODS}

This was a Prospective observational study conducted from 2017 to 2019 in the Department of Radio-diagnosis, Kalinga Institute of Medical Sciences and Bhubaneswar. This study was approved by the Ethical Committee of our institution. The study included 50 singleton pregnancies with suspected IUGR and 50 healthy uncomplicated pregnant women as control cases.

All Singleton pregnancies of gestational age of above 28 weeks with clinically suspected intrauterine growth retardation were included. Multiple fetal gestations or fetuses having major congenital anomalies were excluded.

\section{Method of Collection of Data}

A prospective study of 50 antenatal patients with IUGR and 50 control cases was done after considering the inclusion and exclusion criteria. All the subjects in the study were followed up from first visit up to the time of delivery. Gestational age was confirmed by LMP or by first trimester ultrasound if available. Ultrasound was performed on GE colour Doppler ultrasound scanner with a curvilinear transducer, having a variable frequency of 3.0-5.0 MHZ.

All the subjects were first evaluated by grey scale imaging to assess fetal biparietal diameter, head circumference, abdominal circumference, femur length, amniotic fluid index, head/abdominal circumference ratios in the third trimester of pregnancy i.e. after 28 weeks.

Flow velocity waveforms of the umbilical artery $(\mathrm{UmbA})$ and middle cerebral artery (MCA) were obtained from all the cases and were analyzed. Umbilical artery showing normal wave forms and good diastolic flow (Fig.1 A) and Middle cerebral artery showing normal wave forms and velocimetry (Fig.1 B) are illustrated in Fig.1

Color Doppler UA and MCA were done in standard plane. The umbilical artery (cord) was located using a duplex scanner and will identify as twin parallel white lines in the pool of amniotic fluid. To locate MCA the first step was obtaining plane for BPD measurement. Remaining in same plane probe moved to the base of brain until the sphenoid bone was seen and circle of Willis was located. The MCA conveniently courses along the greater wing of sphenoid and readily seen by Color Doppler imaging. The MCA in the near field was insonated about $1 \mathrm{~cm}$ distal to its origin from the internal carotid artery.

The Pulsatility index (PI), presence or absence of enddiastolic flow and reverse diastolic flow were noted. The UmbA PI was used in progressive absence or end diastolic or reverse diastolic flow (Fig-2 A) as it continues to reflect changes in resistance. The MCA PI/UmbA PI ratio was calculated. In our study a single cutoff value of 1.08 for $\mathrm{MCA} / \mathrm{Umb}$ A PI ratio (cerebral-umbilical ratio) was used, above which velocimetry was considered normal and below which it was considered abnormal, according to reference values. ${ }^{9,10,11}$

\section{Outcome Criteria}

Outcome variables included were birth weight (less than 10th percentile), perinatal death, emergency CS for fetal distress, 5 minute Apgar score less than 7, low birth weight with NICU admissions. Pregnancy was considered to have "Adverse outcome" when any of the following complications were present such as perinatal death, emergency CS for fetal distress and admission to NICU for complications of low birth weight. When the above complications are absent pregnancy outcome will be considered as uneventful or favorable Umbilical artery showing normal wave forms e. The outcome for each pregnancy was observed in NICU and labor ward along with medical records.

\section{STATISTICAL ANALYSIS}

The diagnostic statistics were used to find the diagnostic value of Umb A PI, MCA PI and MCA/UmbA PI in relation to perinatal outcome. The sensitivity, specificity, positive predictive value, negative predictive value and diagnostic accuracy were determined for all Doppler measurement. The statistical software namely Stata-15.1 version was used for the analysis of the data and Microsoft Word and Excel have been used to generate graphs, tables etc.

\section{RESULTS}

\section{Distribution of age of the patients}

All our patients ranged from 20 years to 38 years. Majority of the patients $(58 \%)$ were in $26-30$ years age group. The average age of the patients was 28.16 years. Least number of patients were seen in the age group of more than 30 years.

\section{Distribution of gestational age at doppler examination}

In our study group of 50 antenatal mothers, the gestational age at the time of Doppler examination ranged between

\begin{tabular}{|c|c|c|c|c|c|c|c|c|}
\hline \multirow[t]{2}{*}{ S. $\mathbf{N}$} & \multirow{2}{*}{\multicolumn{2}{|c|}{ Type of wave form }} & \multicolumn{3}{|c|}{ Umbilical artery } & \multicolumn{3}{|c|}{ Middle cerebral artery } \\
\hline & & & PI & $\mathbf{R I}$ & S/D & PI & RI & CPR \\
\hline \multirow[t]{2}{*}{1} & \multirow[t]{2}{*}{ Study group } & Mean & 1.51 & 0.70 & 3.37 & 1.46 & 0.70 & 0.95 \\
\hline & & Standard deviation & 0.8 & 0.136 & 0.16 & 0.13 & 0.13 & 0.39 \\
\hline \multirow[t]{2}{*}{2} & \multirow[t]{2}{*}{ Control group } & Mean & 0.98 & 0.62 & 2.7 & 1.75 & 0.79 & 1.81 \\
\hline & & Standard deviation & 0.18 & 0.07 & 0.55 & 0.37 & 0.09 & 0.47 \\
\hline \multicolumn{3}{|c|}{ Test of significance (P-value) } & $<0.001$ & $<0.001$ & $=0.002$ & $=0.042$ & $<0.001$ & $<0.001$ \\
\hline
\end{tabular}




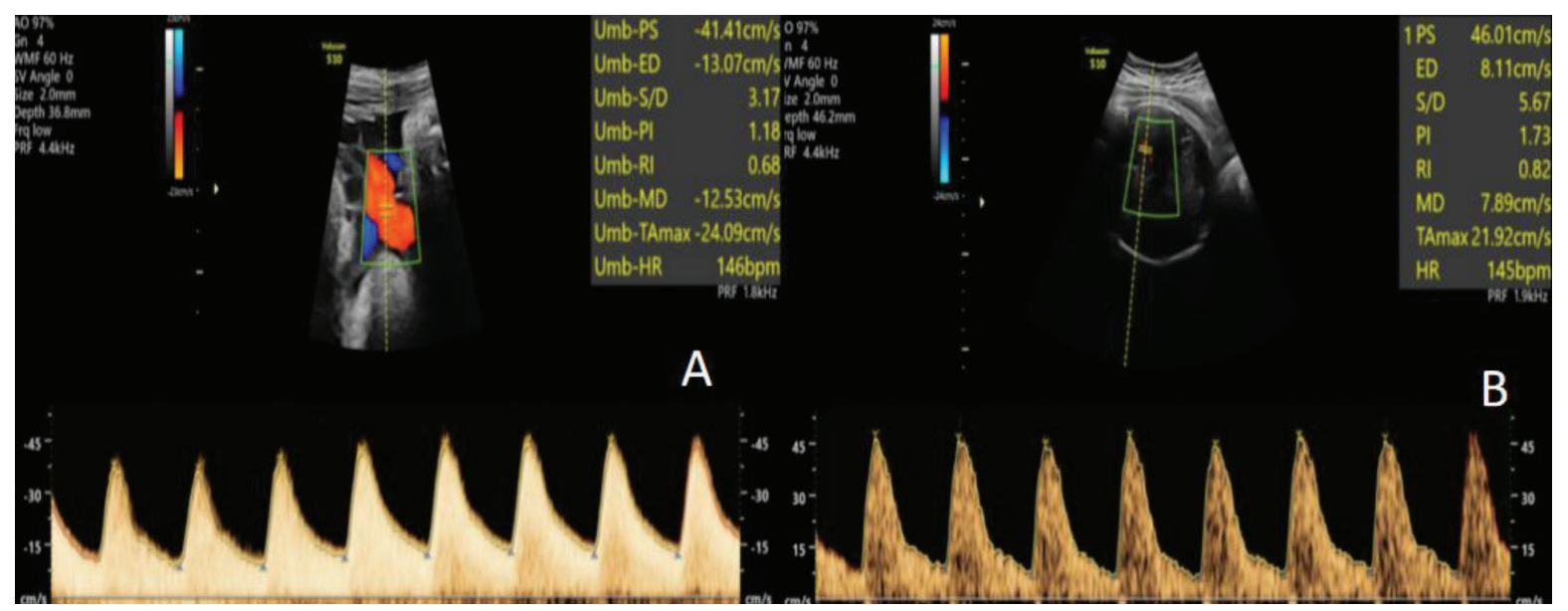

Figure-1: Umbilical artery showing normal wave forms and good diastolic flow.(A) Middle cerebral artery showing normal wave forms and velocimetry. (B)

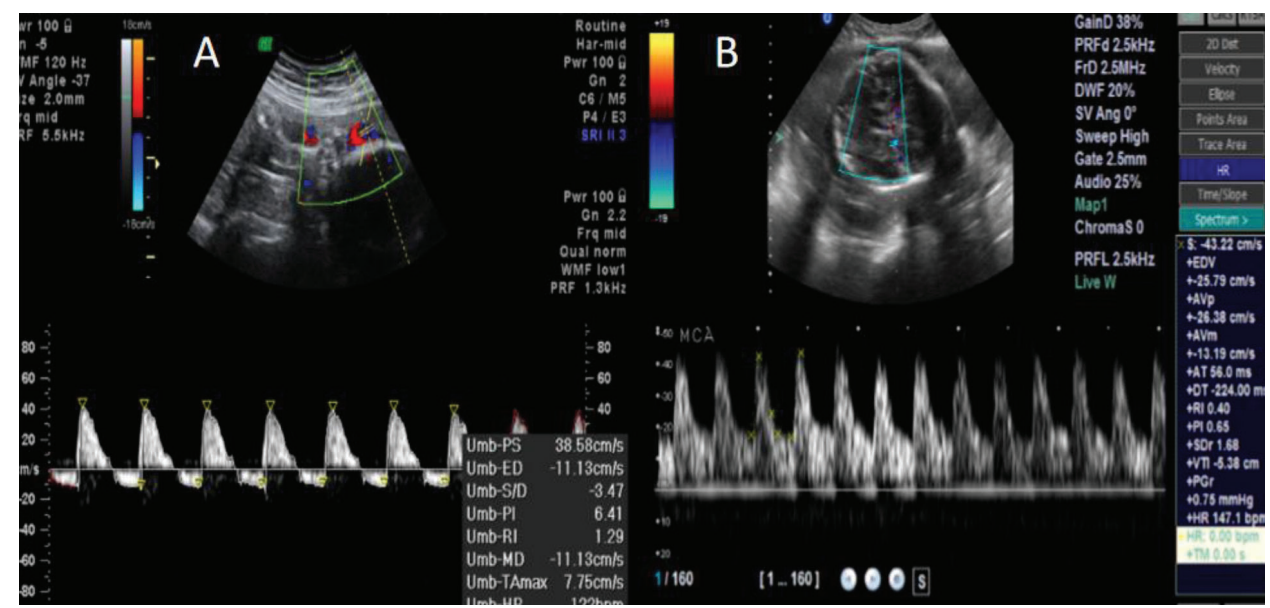

Figure-2: Umbilical artery showing reverse diastolic flow (A). MCA showing continuous forward flow with increased diastolic flow and decreased PI in IUGR fetus (B).

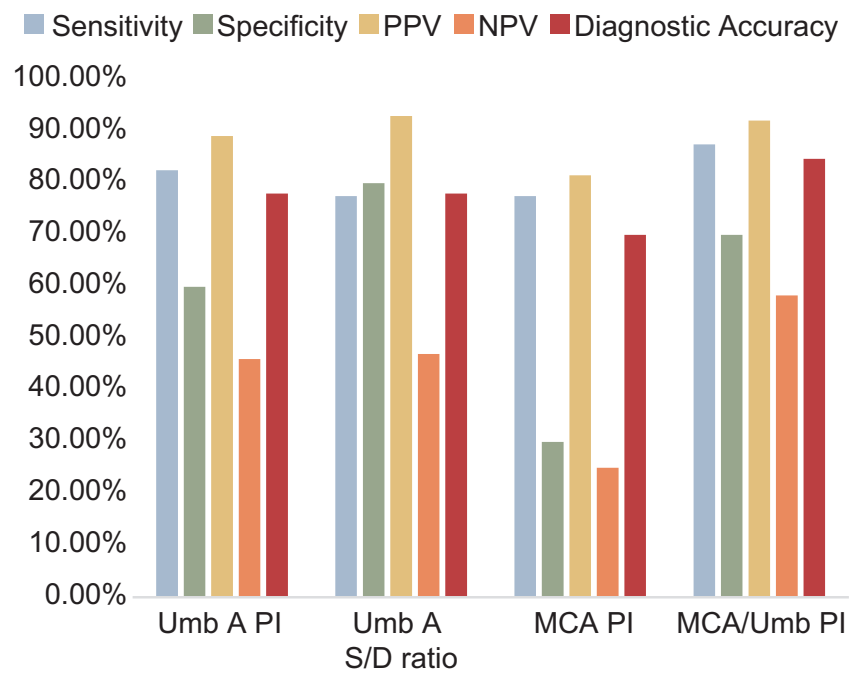

Graph-1: Showing comparison of Doppler indices with adverse perinatal outcome

28- 38 weeks. $70 \%$ of the antenatal mothers examined were between 31-35 weeks of gestation, $18 \%$ between $28-30$ weeks gestation and $12 \%$ with gestational age between $36-40$ weeks. None of the antenatal mothers were examined before 28 weeks of gestational age.
Clinical presentation and indication for doppler study In the study group of 50 antenatal mothers, $50 \%$ of patients had pregnancy-induced hypertension which is the most common cause for IUGR in our series. Only IUGR as the indication for the Doppler study was seen in 24\%. IUGR with anemia complicating pregnancy was found in $20 \%$ of cases. Bad obstetric history was seen in $6 \%$ of cases.

\section{Fetal umbilical artery doppler study}

Umbilical Artery Pulsitility Index (PI): Umbilical artery PI was elevated in 37 patients (74\%) and was normal in 13(26\%) patients.

Umbilical artery end diastolic velocity flow pattern: Three fetuses (6\%) showed absent diastolic flow and 1 fetus (2\%) had reversal of end diastolic umbilical artery flow (Fig.2 A) with the total of 46 fetuses (92\%) having less positive diastolic flow.

Umbilical artery systolic/diastolic (S/D) ratio: 33 out of 50 fetuses (66\%) showed elevated S/D ratio, 17 fetuses (34\%) showed normal S/D ratio.

\section{Fetal middle cerebral artery doppler study}

Middle Cerebral Artery PI: 38 out of 50 fetuses (76\%) had decreased Pulsatility index and 12 fetuses (34\%) had normal Middle cerebral artery Pulsatility index. 
Ratio of middle cerebral artery pi to umbilical artery PI: 40 out of 50 fetuses (80\%) had evidence of redistribution of blood to the brain as evidenced from the ratio of PI in the MCA and umbilical arteries.

Analysis of perinatal outcome: There was one intrauterine death, and 49 live births. One baby died in postnatal day 1 and another baby died in post natal day 3. Of the 47 live births 5 neonates were admitted to NICU, 35 neonates had 5 min APGAR score of less than 7 and 30 babies were born by emergency cesarean section. Mean birth weight at delivery was $2006 \pm 451 \mathrm{gm}$ (2SD). There were 41 neonates with birth weightless than $2.5 \mathrm{~kg}$. 40 fetuses had at least one adverse perinatal outcome. Remaining 10 fetuses had favourable outcome. Absence or reversal of the end diastolic flow velocity was seen in 4 fetuses $(8 \%)$ in our study. There were 3 deaths in whom two cases had absent diastolic flow and one had reverse diastolic flow. The mortality in cases of reverse and absent end diastolic flow was $100 \%$ and $66 \%$ respectively.

\section{Comparison of mean value of doppler indices between study and control group}

In umbilical artery, S/D ratio, PI and RI of IUGR fetuses were significantly higher han that of normal fetuses $(3.37 \pm 0.16$ vs $2.7 \pm 0.55 ; \mathrm{p}<0.01,1.51 \pm 0.8$ vs $0.98 \pm 0.18$; $\mathrm{p}<0.001,0.70 \pm 0.136$ vs $0.62 \pm 0.07 ; \mathrm{p}<0.001)$. In middle cerebral artery, PI of IUGR fetuses was significantly lower than that of normal fetuses $(1.46 \pm 0.13$ vs $1.75 \pm 0.37$; $\mathrm{p}<0.42$ ). Cerebro-placental ratio (PI OF MCA /PI OF UA) of IUGR fetuses was significantly lower than that of normal fetuses $(0.95 \pm 0.39$ vs. $1.89 \pm 0.47 ; \mathrm{p}<0.001$ (Table-1).

\section{DISCUSSION}

Intrauterine growth restriction has multifactorial etiology associated with events occurring along several different pathways. Hence, accurate antenatal diagnosis must conclude whether the fetus is constitutionally small for gestational age or small as a consequence of impaired placental perfusion. Doppler flow velocity analysis can be valuable in solving aforementioned problem, by examining umbilical arteries (fetoplacental circulation) and middle cerebral artery (fetal circulation).

In our study, color Doppler study of 50 pregnant women with suspected IUGR fetuses based on clinical suspicion and grey scale ultrasound examination. 50 normal pregnancies after 28 weeks were taken as healthy control cases. Numerous studies with different techniques and criteria and with varying results have been published in small to large sized population, in high and low risk area. No universally accepted criterion is established for defining abnormal Doppler indices in relation to pregnancy outcome and therefore conflicting observations continue to emerge.

\section{Age Incidence}

We observed that the maximum number of pregnant women were in the age group of 26-30 years (58\%), with a mean age group of 28.16 years. This could be attributed for the increased rate of pregnancies in this age group.

\section{Distribution of gestational age}

In our study all patients underwent Doppler study in third trimester of their pregnancy with maximum of $70 \%$ being investigated between 31 - 35 weeks of gestation. The earliest study was done at 28th week of gestation. Hence most pregnancies were monitored between 31-35 weeks, when the fetus would have begun developing sufficient lung maturity to survive outside the uterus.

\section{Etiology of IUGR}

About $20 \%$ of cases had anemia complicating the pregnancy. Hypertensive disorders were present in maximum of $50 \%$ of pregnancies complicated with IUGR. Bad obstetric history as a cause is seen in $6 \%$ of cases. Twelve patients (24\%) of the study group had no detectable cause for IUGR. Similar findings were seen in Deepti et al ${ }^{12}$ with $50 \%$ cases having pre-eclampsia, 35\% had anemia.

\section{Umbilical artery}

In the present study the umbilical artery PI had a sensitivity of $82.5 \%$. (Graph) According to D. Gramellini et $\mathrm{al}^{13}$, the sensitivity of Umbilical artery PI in predicting perinatal outcome was $64 \%$. Another study by KW Fong et $\mathrm{al}^{14}$ showed the sensitivity of the umbilical artery PI as $58.3 \%$. Umbilical artery was the main vessel used for monitoring high risk pregnancies. This is because umbilical artery represents fetoplacental system and primarily reflects placental resistance. In present study, as there is more number of PIH cases probably this can be attributed for difference in the studies.

\section{Fetal blood circulation and redistribution}

In the present study, fetal middle cerebral artery (MCA) PI had a sensitivity of 77.5\%.(Graph) In a study by Arduini and Rizzo $^{15}$, the sensitivity of MCA PI in predicting perinatal outcome was $68 \%$ and $72.4 \%$ in a study done by K.W Fong et al.

The redistribution calculated from the ratio of the PI of MCA to PI of umbilical artery showed $80 \%$ of the fetuses to redistribute their cardiac output. The sensitivity of MCA PI / UmbA PI of our study is $87.5 \%$ (Graph) in predicting perinatal outcome. These results are slightly higher than that of study done by D Gramellini et $\mathrm{al}^{13}(68 \%)$ and Odibo et $\mathrm{al}^{16}$ (65\%), probably this may be due to small sample size.

\section{Prediction of perinatal outcome}

40 fetuses (80\%) had at least one adverse outcome. Remaining 10 (20\%) fetuses had favorable outcome. There was one intra uterine death showing reversal of umbilical artery diastolic flow in prenatal Doppler study. Out 49 live births, 2 cases died within 3 days of delivery and were showing absent diastolic flow on umbilical artery Doppler study prenatally. The mortality in cases of reversed and absent end diastolic flow was $100 \%$ and $66 \%$ respectively, indicating grave prognosis. 5 neonates (10\%) were admitted to NICU, $35(70 \%)$ neonates had 5 min APGAR score less than 7, and 30 babies (60\%) were born by emergency cesarian section. A study done by Gramellini et $\mathrm{al}^{13}$ showed $55 \%$ cases required emergency caesarian section and another study done by Eslamian et $\mathrm{al}^{17}$ showed $14.4 \%$ of NICU admission which are showing similar results as that of ours.

By using Doppler ultrasound results for analysis, the MCA/ 
UmbA pulsatility index ratio had a higher sensitivity and positive predictive value for predicting the adverse perinatal outcome than the MCA and the UmbA pulsatility indices. Our findings agree with the results of the studies such as Bano $\mathrm{S}$ et $\mathrm{al}^{18}$ (with sensitivity of $83.3 \%$ ) that have shown MCA/UmA PI Doppler ratio to be more useful than UmbA $\mathrm{PI}$ or MCA PI in predicting the adverse outcome.

\section{CONCLUSION}

The umbilical-placental and cerebral vascular beds are directly involved in the hemodynamic adjustments of fetal growth restriction. A Doppler index that reflects both of these areas can be useful for identifying fetuses with increased placental and/or decreased cerebral resistance.

Assessment of both the utero-placental and the feto-placental circulations together is more sensitive to predict to perinatal outcome, than assessment of each alone. In suspected IUGR, cerebro-placentalratio (MCA/UA PI) is a better predictor of adverse perinatal outcome than an abnormal MCA PI or UmbA PI. Best results are obtained when we use MCA/ UmbA PI Ratio, rather than PI values of middle cerebral artery and Umbilical artery separately. Presence of absent/ reversal of diastolic flow in umbilical artery is an ominous sign since it carries a grave prognosis and high mortality. Doppler imaging is of value for monitoring the pregnancy because it can provide indirect evidence of fetal compromise and is known to improve outcomes of high risk pregnancies with intrauterine growth restriction. Hence Doppler evaluation is complementary to all other surveillance modalities.

Limitation of the study: Our study included less number of cases of IUGR of fetuses with evaluation of umbilical and MCA only. Prospective study including more number of cases will help to defined more definite criteria of Doppler indices for predicting adverse perinatal outcome.

\section{Summary}

The sensitivity of Doppler examination of the umbilical artery using PI in predicting perinatal outcome was $82.5 \%$. Doppler studies of the umbilical artery and uterine artery together had a better sensitivity than that of the individual vessels.

The MCA/Umb PI ratio showed more fetuses to redistribute their cardiac output than the abnormal MCA PI or UmbA PI. The cerebro-umbilical ratio provided a better predictor of intrauterine growth restriction and adverse perinatal outcome than either the middle cerebral artery or umbilical artery alone.

\section{REFERENCES}

1. Berkley E, Chauhan SP, Abuhamad A, Society for Maternal-Fetal Medicine Publications Committee. Doppler assessment of the fetus with intrauterine growth restriction. Am J Obstet Gynecol. 2012;206(4):300-8

2. American College of Obstetricians and Gynecologists. ACOG Practice Bulletin No. 204. Obstet Gynecol. 2019;133(2):e97-e109.

3. Sharma D, Shastri S, Sharma P. Intrauterine growth restriction: antenatal and postnatal aspects. Clin Med Insights Pediatr. 2016;10(1):67-83

4. Khanduri S, Chhabra S, Yadav S, Sabharwal T, Chaudhary M, Usmani T, Goyal A, Sharma H. Role of
Color Doppler Flowmetry in Prediction of Intrauterine Growth Retardation in High-Risk Pregnancy. Cureus. 2017;9(11):e1827.

5. Sharbaf FR, Movahed F, Pirjani R, Teimoory N, Shariat M, Farahani Z. Comparison of fetal middle cerebral artery versus umbilical artery color Doppler ultrasound for predicting neonatal outcome in complicated pregnancies with fetal growth restriction. Biomedical Research and Therapy. 2018;5(5):2296-2304

6. G.V.Prasad, Jyothi, Sarvottam. Role of Doppler Study in the Evaluation of Intrauterine Growth Retardation. Journal of Evidence based Medicine and Healthcare 2015;2(6):7266-7275.

7. Figueras F, Gratacos E. Stage-based approach to the management of fetal growth restriction. Prenat Diagn. 2014;34(7):655-9.

8. Royal College of Obstetricians \&Gynaecologists. Small for gestational age fetus: investigation and management. Green-top Guideline. 2013.

9. Merz E (ed): Ultrasonography in Obstetrics and Gynecology.Stuttgart, Thieme, 2005,

10. Acharya G, Wilsgaard T, Berntsen GK, Maltau JM, Kiserud T. Reference ranges for serial measurements of umbilical artery Doppler indices in the second half of pregnancy. Am J Obstet Gynecol. 2005;192(3):937-44.

11. Srikumar S, Debnath J, Ravikumar R, Bandhu HC, Maurya VK. Doppler indices of the umbilical and fetal middle cerebral artery at 18-40 weeks of normal gestation: A pilot study. Med J Armed Forces India. 2017;73(3):232-241.

12. Sharma DD, Chandnani KC. Clinical study of IUGR cases and correlation of Doppler parameters with perinatal outcome. Int J Reprod Contracept Obstet Gynecol 2016;5(3):4290-6.

13. Gramellini D, Folli MC, Raboni S, Vadora E, Merialdi A. Cerebral-umbilical Doppler ratio as a predictor of adverse perinatal outcome. Obstet Gynecol. 1992;79(3):416-20.

14. Fong KW, Ohlsson A, Hannah ME, Grisaru S, Kingdom J, Cohen H, Ryan M, Windrim R, Foster G, Amankwah K. Prediction of perinatal outcome in fetuses suspected to have intrauterine growth restriction: Doppler US study of fetal cerebral, renal, and umbilical arteries. Radiology. 1999;213(3):681-9.

15. Arduini D, Rizzo G. Normal values of Pulsatility Index front fetal vessels: A cross-sectional study on 1556 healthy fetuses. J Perinat Med. 1990;18(3):165-72.

16. Odibo AO, Riddick C, Pare E, Stamilio DM, Macones GA. Cerebroplacental Doppler ratio and adverse perinatal outcomes in intrauterine growth restriction: evaluating the impact of using gestational age-specific reference values. J Ultrasound Med. 2005;24(9):1223-8.

17. Eslamian ZL, Zarean E, Moshfeghi M, Heidari Z. Evaluation of the predictive value of fetal Doppler ultrasound for neonatal outcome from the 36th week of pregnancy. J Res Med Sci. 2018;23(5):13.

18. Bano S, Chaudhary V, Pande S, Mehta V L, Sharma A K. Color doppler evaluation of cerebral-umbilical pulsatility ratio and its usefulness in the diagnosis of intrauterine growth retardation and prediction of adverse perinatal outcome. Indian J Radiol Imaging 2010;20(2):20-5

\section{Source of Support: Nil; Conflict of Interest: None}

Submitted: 27-11-2019; Accepted: 31-12-2019; Published online: 28-02-2020 